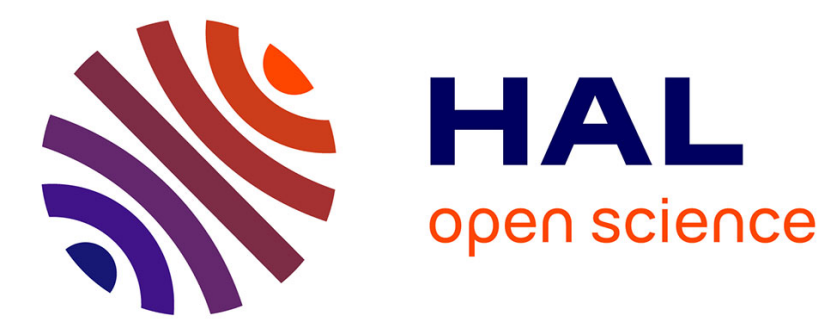

\title{
Fostering the Collaboration among Healthcare Stakeholders with ICF in Clinical Practice: EasyICF
}

Daniele Spoladore, Atieh Mahroo, Marco Sacco

\section{To cite this version:}

Daniele Spoladore, Atieh Mahroo, Marco Sacco. Fostering the Collaboration among Healthcare Stakeholders with ICF in Clinical Practice: EasyICF. 22nd Working Conference on Virtual Enterprises (PRO-VE 2021), Nov 2021, Saint-Etienne, France. pp.623-631, 10.1007/978-3-030-85969-5_58 . emse-03346761

\section{HAL Id: emse-03346761 https://hal-emse.ccsd.cnrs.fr/emse-03346761}

Submitted on 25 Nov 2021

HAL is a multi-disciplinary open access archive for the deposit and dissemination of scientific research documents, whether they are published or not. The documents may come from teaching and research institutions in France or abroad, or from public or private research centers.
L'archive ouverte pluridisciplinaire HAL, est destinée au dépôt et à la diffusion de documents scientifiques de niveau recherche, publiés ou non, émanant des établissements d'enseignement et de recherche français ou étrangers, des laboratoires publics ou privés. 


\title{
Fostering the Collaboration among Healthcare Stakeholders with ICF in Clinical Practice: EasyICF
}

\author{
Daniele Spoladore ${ }^{1,2}$, Atieh Mahroo $^{1}$ and Marco Sacco ${ }^{1}$ \\ ${ }^{1}$ Institute of Intelligent Industrial Technologies and Systems for Advanced Manufacturing \\ (STIIMA), National Research Council of Italy (CNR), 23900 Lecco, Italy \\ ${ }^{2}$ Department of Pure and Applied Sciences, Computer Science Division, Insubria \\ University, 21100 Varese, Italy, \\ \{daniele.spoladore, atieh.mahroo, marco.sacco \}@stiima.cnr.it
}

\begin{abstract}
The International Classification of Functioning, Disability and Health (ICF) is a standard framework organized as a top-down taxonomy composed of categories allowing to describe a person's functioning within an environment (physical and social context). ICF aims at helping different clinical professionals and health stakeholders to adopt a common conceptual basis and standard definitions for the description of disabilities and impairments, thus easing the collaboration and clinical information exchange among different actors. ICF is not universally adopted in clinical practice: this is caused mainly by a lack of understanding of ICF and of the operative knowledge to qualify impairments. This work introduces EasyICF, an application designed to facilitate the use of ICF in defining wheelchair users' health conditions for vocational therapists and clinical personnel. EasyICF takes into account the restrictions foreseen in the framework and is designed to support the definition of ICF categories for clinicians with little or no knowledge of the classification and its structure. The application was developed with the help of medical personnel and physical therapists, in a collaborative effort to develop a tool for daily clinical practice in the context of Return to Work.
\end{abstract}

Keywords: ICF, return to work, clinical stakeholders collaboration

\section{Introduction}

This International Classification of Functioning, Disability and Health (ICF) is a World Health Organization (WHO) standard classification aimed at providing a description of an individual's health condition, taking into account functional aspects, body structures, activities and environmental factors. ICF serves as a conceptual standard basis for the definition of disabilities and impairments and focuses on the person and the context surrounding him/her: the functioning of the individual in specific domains derives from the interactions between the person's health condition and the physical and social environment in which he/she acts [1]. The classification is 
organized into four main components, which should be completed by a fifth component that - to date - has not been developed yet (Personal factors):

- Body functions describe the psychophysiological functions of the body;

- Body structures identify the anatomical parts of the human body and their components;

- Activities and participation describe, respectively, the execution of tasks in life and the individual's involvement in specific situations;

- Environmental factors provide the means to describe the physical and social context in which the person lives, identifying facilitators and barriers.

Each component involves different health domains and is structured into categories, which constitute the atoms of the classification. The categories are identified by a prefix letter ("b" for Body functions, "s" for Body structures, "e" for Environmental factors, "d" for Activities and Participation) and are further detailed by adding digits: the longer is the number of the digits, the more detailed is the category; contrariwise, the lesser is the number of the digits following the letter, the more general is the health-concept represented by the category. Categories can therefore be structured in a hierarchical taxonomy in which the categories with fewer digits contain those with more digits (Figure 1 illustrates this concept with an excerpt of ICF).

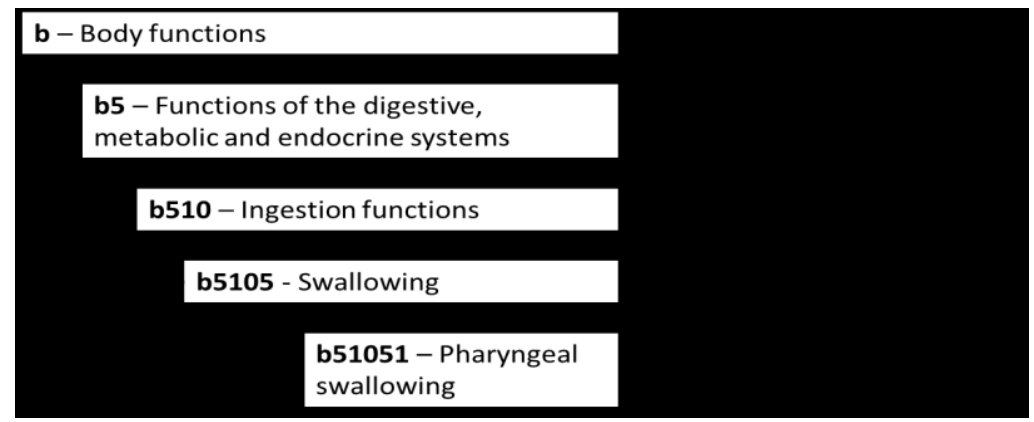

Fig 1. An excerpt of the ICF classification highlighting its hierarchical structure.

The total number of categories amounts to 1454 and they allow the conceptualization of several domains involved in the definition of an individual's functioning and disability. Each category is provided with a text providing unambiguous definitions of the item (e.g.: b51051 - Pharyngeal swallowing is defined as "Function of clearing substances through the pharynx at an appropriate rate and speed"). The definition also provides examples of what can be considered within the range of the category (inclusion) and what is excluded (e.g.: b51051 has as "Inclusions: functions of production and quality of voice; functions of phonation, pitch, loudness and other qualities of voice; impairments such as aphonia, dysphonia, hoarseness, hypernasality and hyponasality", while indicates as "Exclusions: b167 Mental functions of language; b320 - Articulation functions; b340 - Alternative vocalization functions").

To quantify the severity of impairment, each item is associated with one (or more) qualifier(s), integer numbers following the item and separated from it by a "." (e.g.: b51051.3): the number of qualifiers an item can accept is determined by its type [2]. 
For instance, Body functions accepts only one qualifier (called "generic qualifier") whose values range from 0 to 4 ( 0 = "no impairment", 1 = "mild", 2 = "moderate", 3 = "severe", 4 = "complete"), with the addition of 8 (qualifier "not specified") and 9 (qualifier "not applicable"). However, the component Body structures accepts up to three qualifiers (the second indicating the nature of the change happened in a body structure, the third describing the location of the impairment in the body, all using integers from 0 to 9 to indicate different causes and locations of the impairment), while Environmental factors makes use of the generic qualifier, preceded by "+" to indicate a facilitator (e.g.: e5400.3 indicates a very relevant facilitator in "Transportation services") or without sign to indicate a barrier (e.g.: e410.1 suggests a mild barrier in the "Individual attitudes of immediate family members"). Activities and participation dedicate the first and fourth qualifier to describe the performance, which is the activity conducted in the individual's current environment, while the second and third describe the capacity, which identifies a person's ability to execute a task in a "standardized" environment (like a laboratory or clinical setting); with/without assistance indicates whether or not the individual executing the task is evaluated in his/her performance or capacity while using an assistive device or personal assistant (e.g.: glasses, wheelchair, etc.) [3]. Table 1 summarizes the possible qualifiers applicable to ICF items and their meanings.

Table 1. A summary of possible qualifiers applicable for each ICF component.

\begin{tabular}{ccccc}
\hline Component & $1^{\text {st }}$ qual. & $2^{\text {nd }}$ qual. & $3^{\text {rd }}$ qual. & $4^{\text {th }}$ qual. \\
\hline $\mathrm{b}$ & Generic qualifier & - & - & - \\
$\mathrm{s}$ & Generic qualifier & Nature of the change & $\begin{array}{c}\text { Localization of } \\
\text { the impairment }\end{array}$ & - \\
$\mathrm{d}$ & $\begin{array}{c}\text { Performance (with } \\
\text { assistance) }\end{array}$ & $\begin{array}{c}\text { Capacity (without } \\
\text { assistance) }\end{array}$ & $\begin{array}{c}\text { Capacity (with } \\
\text { assistance) }\end{array}$ & $\begin{array}{c}\text { Performance } \\
\text { (without } \\
\text { assistance) }\end{array}$ \\
$\mathrm{e}$ & $\begin{array}{c}\text { Generic qualifier (+ } \\
\text { for facilitators) }\end{array}$ & - & - & - \\
\hline
\end{tabular}

Together with a conceptual framework for the description of disability, ICF was developed as a tool to ease the communication among health-stakeholders (clinicians, therapists, bioengineers, designers of assistive solutions, etc.), since it provides an unambiguous vocabulary. During the early 2000 s, ICF gathered a lot of attention and was progressively adopted in many health-related fields (e.g.: frameworks for the rehabilitation [4, 5], Electronic Health Records [6], assessing factors hindering the social participation of people with disabilities [7], etc.).

However, although some solid examples of ICF applications exist, the adoption of ICF in clinical practice is still limited. ICF is a very wide classification, and there exist some barriers in its adoption that prevent healthcare professionals in using the standard as a common language. In this work, we propose to overcome some of the barriers by using an application - namely EasyICF - that allows clinical personnel to build individuals' health condition descriptions using the ICF. We argue that the possibility to rely on an application can significantly reduce the need of specialized training and can support healthcare professionals in selecting categories and qualifiers. Therefore, we argue that to foster cooperation among stakeholders the use of ICF as a "common language" can be made easier by relying on digital tools that 
can help clinicians and health professionals to overcome some of barriers in the adoption of this standard.

The reminder of this paper is organized as follows: we investigate some of the major barriers hindering ICF adoption in clinical practice (Sect. 2). Within the context of Return to Work (RTW) [8], we developed an application aimed at providing a possible solution (Sect. 3), for which a brief overview of the underlying technology is provided (Sect. 4). Finally, Conclusions summarize the main outcomes of this work.

\section{Major Issues in the Adoption of ICF in Clinical Practice}

Although ICF is universally recognized as a standard able to foster professional and non-professional health stakeholders' information exchange, its actual application in clinical practice and rehabilitation is very limited. The first well-known problem is related to the adoption of ICF in its complete form: as pointed out in many studies [9] ICF structure does not allow for an agile adoption of all its categories, and therefore Core sets - subsets of ICF categories providing a comprehensive description of specific health issues - were developed to ease the adoption of ICF in clinical practice [10]. Nevertheless, the use of the framework within clinical contexts remains limited: different studies have analyzed the reasons behind the underutilization of ICF, which can be summarized in:

a. Need of specific training to increase the actual knowledge of ICF and its underlying conceptual model [11];

b. Significant difficulties in the implementation in some fields, including rehabilitation [12] and underestimation of the time and resources necessary for the implementation [13];

c. Lack of standard methods for the selection of the proper qualifiers [14];

d. The necessity to adapt ICF to the individual territories and clinical realities adopting it into their clinical practice [9];

These problems may significantly hinder the adoption of ICF in clinical and rehabilitation contexts, thus undermining the cooperation among different health professionals and stakeholders. In particular, for multi-domain interventions in fields requiring the collaboration of different professionals (e.g.: Ambient Assisted Living [15], Continuity of Care [16], RTW, etc.), the issues a. and b. may result in the inability to deliver tailored solutions to patients.

\section{EasyICF: Fostering the Adoption of ICF in RTW Contexts}

EasyICF was developed to tackle those issues related to the necessity of training to increase the actual knowledge of ICF and to reduce the difficult implementation of ICF-based solutions in clinical practice. This problem emerged in the context of research project dedicated to RTW, but its underlying questions and findings are common for any discipline that foresee the adoption of ICF at different levels. 


\subsection{The Rientr@Project}

EasyICF is one of the results of an Italian research project aimed at fostering the RTW of novice wheelchair users (WU) [8]: the Rientr@ project, promoted by the Italian National Institute for Insurance against Accidents at Work (INAIL). In Rientr@, the patients follow a RTW evaluation process that foresees a multidisciplinary evaluation of their abilities to understand the extent of their disability and to infer which professions they can still perform according to their health condition. In this context, many health professionals are involved: clinicians, physical therapists, biomedical engineers and vocational therapists need to cooperate to tailor the RTW process to the individual's specific needs - therefore they need to "speak the same language". Working in close collaboration with these professionals from INAIL, we were able to identify that problems related to actual knowledge of ICF and time-related issues hindered the adoption of the ICF as a tool in clinical practice, although some training on the ICF was already administered to INAIL personnel. Leveraging on meetings and test cases (i.e. a set of real health conditions of WUs) and adopting a methodology derived from collaborative engineering of knowledge bases [17], INAIL personnel was able to identify the problems described above, thus allowing us to developed a tailored solution in the form of an application: in fact, an application based on ICF serves as a promising solution to reduce the time necessary to formalize a health condition, and to minimize the necessity of training. The application, named EasyICF, was based on those ICF Core sets, which correspond to the main causes that force persons on a wheelchair (Spinal Cord Injury, Traumatic Brain Injury, Stroke); the Core set for Vocational Rehabilitation was added to better address the description of concepts related to WUs' work reintegration.

\subsection{EasyICF Development}

EasyICF was developed as an application that helps clinical professionals in inserting and/or updating a WU's health condition, by selecting the appropriate ICF categories from those composing the Core sets and guiding the clinicians in selecting the appropriate code. Considering the specific case of RTW, we decided to focus our attention on the Body functions component and Activities and participation (offering the possibility to insert the qualifiers for performance and capacity both with assistance, considering that the wheelchair is necessary for performing any task in any environment).

EasyICF is designed and developed exploiting Java programming language Standard Edition and Swing toolkit for Graphical User Interface components. This software is designed to be a standalone application, easily installed on computer platforms where health stakeholders are able to insert and/or modify the WU's health condition - thus, updating and manipulating the information on the ICF. Due to the complex structure of ICF, compiling its categories for each patient is an involute procedure that requires a learning and training phase for the health professionals. However, EasyICF can provide a simple and easy-to-use interface for health stakeholders with limited or no knowledge of ICF and helps them in compiling the ICF codes with minimum time and effort. The application provides step-by-step 
guidelines and instructions on choosing the correct ICF code and proper qualifiers while hiding the complexity of the ICF structure from the users. It is also capable of checking the quality of data inserted and warning the user in case of incorporating unacceptable values.

\subsection{Use Case: Inserting a New Health Condition for a Wheelchair User}

The EasyICF application consists of a set of sequential windows displaying information from ICF in which the user is guided and supervised to compile the correct ICF codes for each patient. The process of inserting the health condition of a WU initiates where EasyICF displays the list of WUs that have been saved within a database (in this case, a semantic database as described in [18]). Health professional can select the WU for which they want to insert or modify the health condition (Fig. 2). EasyICF then invites the health professional to choose the proper ICF Core set which coincides with the main reason that forced the person on a wheelchair, (spinal cord injury, traumatic brain injury, or stroke, as illustrated in Fig. 2).

After choosing the proper core set, the application provides a list of ICF categories - which compose the selected Core set - together with their names and complete descriptions, and for each code it highlights inclusions and exclusions (Fig. 3).

Finally, EasyICF helps the operator in defining the qualifiers for all the ICF codes chosen in the previous step with hints on acceptable values and data quality checks (according to Table 1) to ensure the inserted qualifier is a valid ICF qualifier digit (Fig. 4). In the end, all the ICF codes and associated qualifiers are stored in a database - hence the WU's health condition is inserted.

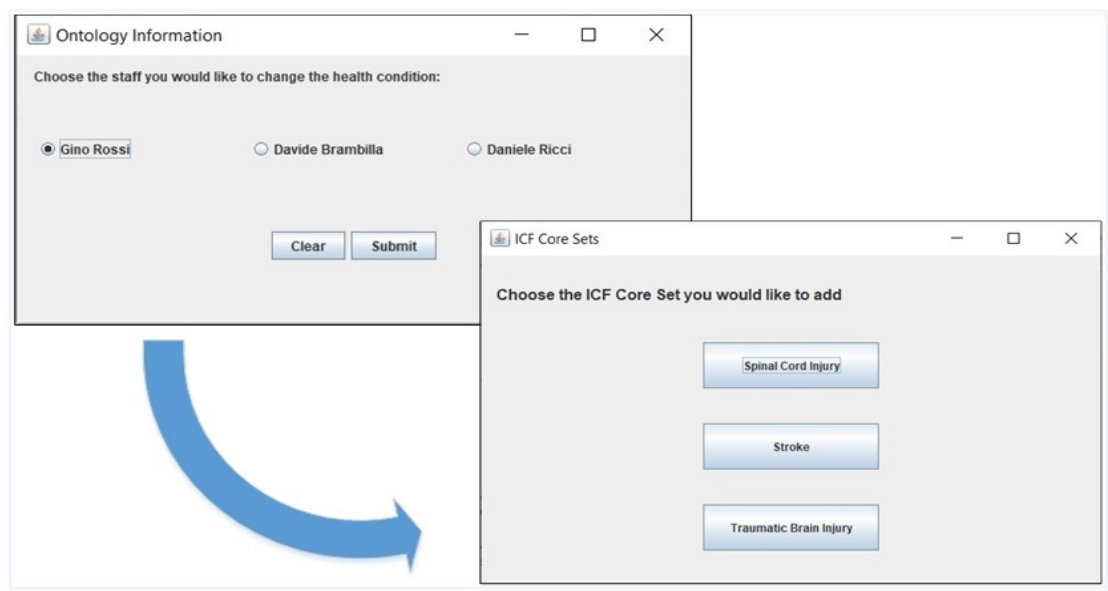

Fig.2 Screenshots of EasyICF to choose the WU (left) and the ICF Core set (right) corresponding to the main cause of WU's impairment.

The application proposes a description of the category to be selected and highlights the inclusion and exclusion, thus helping the compiler (i.e. a health professional) to get a comprehensive view on the definition of the category. Moreover, by selecting a specific category that excludes others, the compiler can significantly reduce the time he/she needs to model a health condition, as the main references and indications to 
use the categories are provided by the application. In this way, the personnel adopting ICF can increase their level of confidence in using the ICF as a tool, thus reducing the necessity of intensive training to get a comprehensive grasp on the classification (as underlined in the first problem reported in Sect. 2).

Also, by allowing clinical personnel to select the Core set adopted as a reference to model a health condition, the risks connected to incurring in a time-consuming activity for health condition modelling are reduced (second problem proposed in Sect. 2). As other Core sets can be added to EasyICF, this feature can also help mitigating the issues related to ICF adoption in particular fields - such as rehabilitation - by using specific Core sets describing the problem(s) that need to be treated.

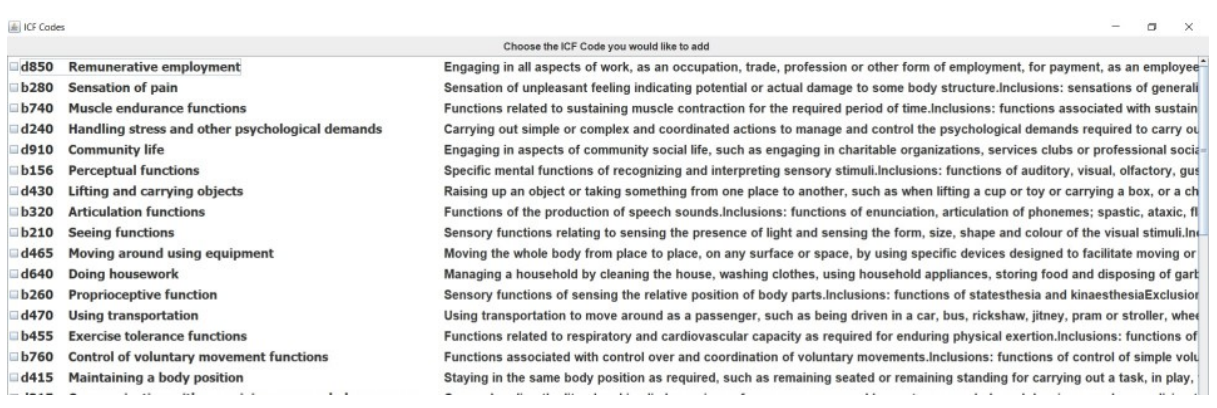

Fig. 3. Screenshot of EasyICF displaying the ICF categories, their names, and complete descriptions to help the operator selecting all the codes to describe the WU's health condition.

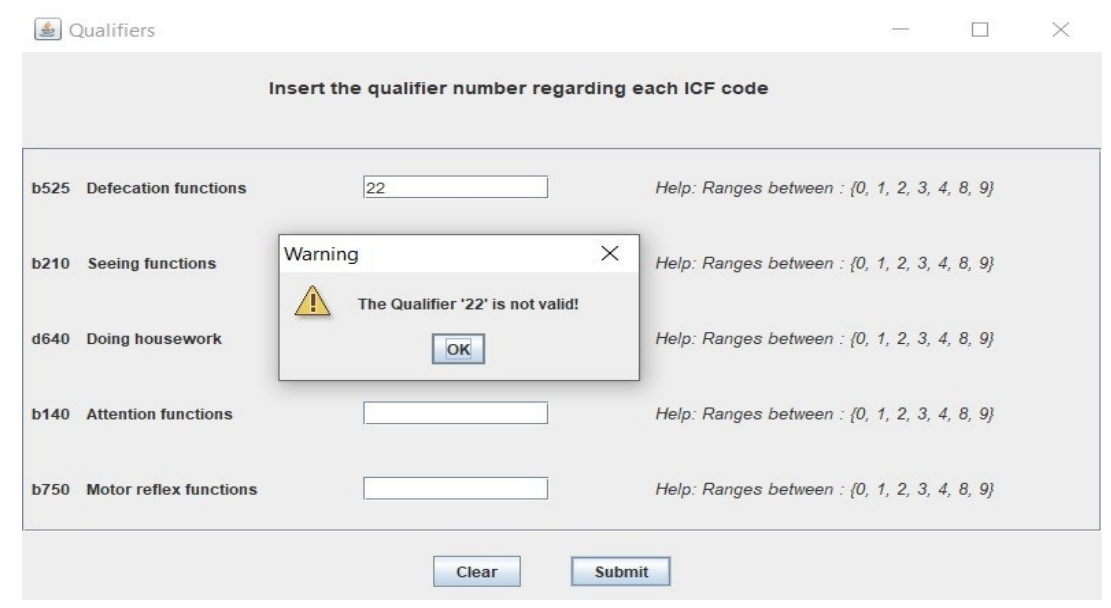

Fig. 4. A screenshot of EasyICF illustrating checks on qualifiers' insertion to guide the operator in the correct compiling of WU's health condition. 


\section{Conclusion and future works}

EasyICF makes a universal environment that can be exploited by different clinical stakeholders who are not necessarily experts in ICF: the possibility to rely on the same classification to exchange health-related information can enhance RTW services provided to WUs through the active collaboration among various healthcare organizations. The application presented is still in its prototypical form and needs to be validated by experts: in particular, to support the hypothesis that EasyICF is able to reduce the time necessary to model a WU's health condition and to reduce the necessity of ICF-specific training sessions, the application will be delivered to social workers (with a very basic knowledge of ICF) involved in the RTW process of novice WUs to be assessed. Although EasyICF was developed specifically for RTW [18], its core idea can be extended to other ICF Core sets to help health stakeholders in describing several health conditions.

From a technical perspective, EasyICF prototype involves only the Body functions and Activities and participation components due to the necessity to describe the functioning of an individual in his/her real context: however, the knowledge base of ICF categories underlying the application can easily be extended to include the other two components, thus providing a more detailed description. In this regard, future works foresee the addition of Environmental factors categories to identify also social and/or physical facilitators and barriers in the working context in which WUs should be inserted: in this way, vocational therapists could exploit the application also to plan specific interventions dedicated to tackle environmental issues: also, this feature would allow to start tackling the problem of adapting ICF to the specific territories (as underlined in Sect. 2).

\section{References}

1. Stucki, G., Kostanjsek, N., Cieza, A.: The International Classification of Functioning, Disability and Health: A Tool to Classify and Measure Functioning. Handbook of Disease Burdens and Quality of Life Measures Preedy VR, Watson RR, eds. New York, NY: Springer New York. 1, 34 (2010).

2. Bruyère, S.M., Van Looy, S.A., Peterson, D.B.: The international classification of functioning, disability and health: Contemporary literature overview. Rehabilitation Psychology. 50, 113 (2005).

3. Organization, W.H., others: A practical manual for using the International Classification of Functioning, Disability and Health (ICF). Geneva: WHO. (2013).

4. Lexell, J., Brogårdh, C.: The use of ICF in the neurorehabilitation process. NeuroRehabilitation. 36, 5-9 (2015).

5. Stucki, G., Ewert, T., Cieza, A.: Value and application of the ICF in rehabilitation medicine. Disability and rehabilitation. 24, 932-938 (2002).

6. Maritz, R., Aronsky, D., Prodinger, B.: The International Classification of Functioning, Disability and Health (ICF) in electronic health records: a systematic literature review. Applied clinical informatics. 8, 964 (2017).

7. Rimmer, J.H.: Use of the ICF in identifying factors that impact participation in physical activity/rehabilitation among people with disabilities. Disability and rehabilitation. 28, 1087-1095 (2006). 
8. Spoladore, D.: Ontology-based decision support systems for health data management to support collaboration in ambient assisted living and work reintegration. In: Working Conference on Virtual Enterprises. pp. 341-352. Springer (2017).

9. Maini, M., Nocentini, U., Prevedini, A., Giardini, A., Muscolo, E.: An Italian experience in the ICF implementation in rehabilitation: preliminary theoretical and practical considerations. Disability and rehabilitation. 30, 1146-1152 (2008).

10. Yen, T.-H., Liou, T.-H., Chang, K.-H., Wu, N.-N., Chou, L.-C., Chen, H.-C.: Systematic review of ICF core set from 2001 to 2012. Disability and rehabilitation. 36, 177-184 (2014).

11. Pernambuco, A.P., Lana, R. de C., Polese, J.C.: Knowledge and use of the ICF in clinical practice by physiotherapists and occupational therapists of Minas Gerais. Fisioterapia e Pesquisa. 25, 134-142 (2018).

12. Escorpizo, R., Stucki, G., Cieza, A., Davis, K., Stumbo, T., Riddle, D.L.: Creating an interface between the International Classification of Functioning, Disability and Health and physical therapist practice. Physical therapy. 90, 1053-1063 (2010).

13. Wiegand, N.M., Belting, J., Fekete, C., Gutenbrunner, C., Reinhardt, J.D.: All talk, no action?: the global diffusion and clinical implementation of the international classification of functioning, disability, and health. American Journal of Physical Medicine \& Rehabilitation. 91, 550-560 (2012).

14. Rauch, A., Cieza, A., Stucki, G.: How to apply the International Classification of Functioning, Disability and Health (ICF) for rehabilitation management in clinical practice. European journal of physical and rehabilitation medicine. 44, 329-342 (2008).

15. Spoladore, D., Mahroo, A., Trombetta, A., Sacco, M.: DOMUS: a domestic ontology managed ubiquitous system. Journal of Ambient Intelligence and Humanized Computing. 1-16 (2021).

16. Spoladore, D., Arlati, S., Colombo, V., Modoni, G., Sacco, M.: A Semantic-Enabled Smart Home for AAL and Continuity of Care. In: IoT in Healthcare and Ambient Assisted Living. pp. 343-371. Springer (2021).

17. Spoladore, D., Pessot, E.: Collaborative Ontology Engineering Methodologies for the Development of Decision Support Systems: Case Studies in the Healthcare Domain. Electronics. 10, 1060 (2021).

18. Arlati, S., Spoladore, D., Mottura, S., Zangiacomi, A., Ferrigno, G., Sacchetti, R., Sacco, M.: Analysis for the design of a novel integrated framework for the return to work of wheelchair users. Work. 61, 603-625 (2018). 